\title{
Six Concepts for Brain-Friendly Leadership: Leading Your Organization Smart and Easy
}

\author{
Sanghee Yeon, Dan Shepherd \\ Continuing Education, Defense Language Institute, Monterey, California \& Department of Education, Missouri Western State University, St. \\ Joseph, the United States
}

Email address:

shyeon@gmail.com (S. Yeon)

To cite this article:

Sanghee Yeon, Dan Shepherd. Six Concepts for Brain-Friendly Leadership: Leading Your Organization Smart and Easy. American Journal of Management Science and Engineering. Vol. 6, No. 5, 2021, pp. 137-141. doi: 10.11648/j.ajmse.20210605.11

Received: April 28, 2021; Accepted: May 15, 2021; Published: October 30, 2021

\begin{abstract}
Employees often comment that they work many hours a day but see little beneficial impact. These people possess adequate intelligence and work diligently, often to the point of losing sleep in favor of additional work time. Researchers have long known that sleep is vital to mood maintenance and body wellness. Does this brain-centered issue have implications for work efficiency, and do we as leaders need to know this to better lead? Recent improvements in technology have provided additional knowledge on how the brain functions. These discoveries enable researchers to see how a person processes information and which factors - sleep, for example - influence how well that processing occurs. However, it seems that these beneficial efforts in cognitive psychology and neuroscience are not applied effectively among the work force. Hence, this paper will connect six foundational concepts, from neuroscience and cognitive psychology (i.e., positive environment, movement, rest, non-monetary reward, repetition, and using gut feelings) to the field of leadership in effort to augment the emphasis of this burgeoning science on that field of study. These six concepts are not at all difficult to achieve in the workplace. Smart leaders are encouraged to take these small steps toward fostering a brain friendly workplace.
\end{abstract}

Keywords: Leadership Development, Brain-Based Leadership, Cognitive Psychology, Neuroscience

\section{Introduction}

Employees often comment that they work many hours a day but see little beneficial impact on work sometimes. These sincere employees possess adequate intelligence and work diligently, often to the point of losing sleep for additional work time. This in itself may be the issue. Researchers have long known that sleep is vital to mood maintenance and body wellness; does this brain-centered issue have any implication for work efficiency? Do we, as leaders, need to know this to lead an organization better? Recent improvements in modern technology (such as functional magnetic resonance imaging (FMRI), Electroencephalography (EEG), etc.) have provided greater knowledge of how the brain functions. These devices enable researchers to see how a person processes information and what factors - sleep, for example - influence how well that processing occurs (e.g., [16]). However, it seems that these beneficial efforts in cognitive psychology and neurology are not applied well in the workforce. When leaders know more about how the brain works, they will become more efficient and effective in guiding their employees. This article endeavors to connect six foundational concepts from neuroscience and cognitive psychology (positive environment, movement, rest, non-monetary reward, repetition, and using gut feelings) to the field of leadership in an effort to augment the emphasis of this burgeoning science on that crucial field of study.

\section{Positive Environment}

People state a clear preference for pleasant surroundings, but is there real, research-based evidence to support that having a pleasant environment actually promotes productivity? The answer is a resounding "Yes!" The anatomical proof, proof that is apparently hard-wired into individual minds, of this assertion starts with brain neurons themselves. Neurons are cells which transmit and process information by electro-chemical signals. Neurotransmitters are chemicals which increase the effectiveness and efficiency of communication among neurons. Dopamine, one of the 
major neurotransmitters, helps regulate the feeling of pleasure and is strongly associated with a person's emotional state. When an employee, for example, perceives that $\mathrm{s} / \mathrm{he}$ is having a pleasant conversation or working in a positive environment, the level of dopamine increases measurably. In addition to emotional states, dopamine is also connected with and appears to augment a person's alertness, perception, and memory [11]. In other words, positive emotions trigger dopamine's release; these increased neurotransmitter levels make employees alert and energized. When this neurotransmitter is abundantly available in their brain, then their productivity and creativity increase.

Of course, there are also negative emotions. When negative emotions are triggered, a different brain hormone is released. This chemical - cortisol - causes a much different reaction in the body, initiating a person's entrance into what is commonly referred to as "survival mode." This biological state of agitation and sensitivity to stress is the opposite of what is needed for optimal thinking and working. Sustained stress further increases the cortisol level, which in turn inhibits the hippocampus or memory center from functioning fully. When cortisol is prominent in a body system, brain activities are significantly hampered [12]. In addition, when negative emotions are triggered, the amygdala, which is related to the regulation of emotion and memory, is overly stimulated. Position emission tomography (PET) scans show that when this happens, information cannot go through the brain's memory circuits efficiently. This physiological response limits full access to the memory area. As a result, creative and productive work is not accomplished [20].

One example in an organization where negative emotions increase is when a leader wants to make even minor changes in the work environment or expectations. These unanticipated changes often result in unpleasant and negative experiences to employees. According to Gordon and Williams [7], all human actions can be placed into two distinct categories: Maximizing reward and minimizing threat. Simply put, most workplace changes are considered as threats, and employee brains immediately enter the often-mentioned "fight or flight" mode. To describe this moment physiologically, the limbic system identifies a threat and releases hormones such as epinephrine to prepare the body to handle the conflict. As a result, while the brain is making these stress-related accommodations, other cognitive functions are necessarily hindered, and thinking just becomes harder.

Given this, what can a leader do to make these experiences less stressful? Research recommends several possible leadership interventions: highlighting or identifying the positive benefits of the change, giving employees opportunities to ask questions, and listening actively to their concerns. Just offering question and answer sessions (i.e., open communication) reduces stress and lowers the threat level [18]. Conversely, sudden meetings and unexpected calls are definitely negative/stress-producing activities. Previously announced meetings give employees time to prepare themselves for change, which makes for a less stressful work environment.
Another action a brain-sensitive leader can take to foster a positive environment in the workplace is to be positive him/herself. Working with people who are perceived as not likable is patently difficult. Once an employee perceives someone else as a possible opponent, his or her logical thinking about that person is significantly impeded. When this happens within the limbic system, the threat mode becomes the "default setting," until a positive cue intervenes. When a person encounters an unpleasant individual, his or her cortisol level rises, and usual blood flow to prefrontal cortex is reduced [12]. Since this area of the brain controls logical decision-making, employees can often react poorly under the influence of increased stress.

Leaders, though, can take impactful action to create a positive mindset within the workplace. For example, positive, smiling leaders are truly, according to current brain research, contagious. Humans and other primates possess "mirror neurons." These interesting brain cells respond identically both when person does something and when a person observes that same action performed by another. When an employee encounters a smiling supervisor, s/he will tend to smile in return. This mirroring is hard-wired into the brain [9]. Conversely, if a leader does not control his or her emotions, employees will be less apt to control theirs as well. Therefore, promoting a positive social environment starts with the leader. Smiling is a necessary first start. Friendly small talk around the water cooler is another worthwhile environment-improving behavior, and the leader can also make a more positive, sociable environment by setting up informal meetings or by welcoming time to share personal stories.

The promotion of fairness is another way to increase the positive environment within the workplace. For a leader, fairness among employees is paramount. Research shows that people prefer fairness even over increased salary. Employees deeply resist inequality; this insistence on being treated fairly is rooted deep in the human brain. When a person feels s/he has been treated unfairly, the threat level dramatically increases. This value is so inherent in the race that individuals do not even like to observe the unfair treatment of others, even strangers they may not know. People are willing to sacrifice their own gain in order to prevent another person's unfair treatment [17]. On the other hand, when people experience fairness, their reward circuits are activated, and they feel a sense of happiness. If unfairness goes unpunished, people's negative emotions are aroused [9]. Again, when this happens, the limbic system becomes supercharged, and the ability to think logically is significantly reduced, which making work more unproductive. A brain-based leader ensures the promotion of fairness throughout the workplace.

\section{Movement}

Researchers have determined that our prehistoric ancestors moved about 12 miles a day [12]. They moved to gather food and to escape from wild animals' pursuit. As a result, the 
modern human brain is pre-wired for frequent movement. Of course, the benefits of physical activity are well-documented. Working out physically prevents diseases, promotes a healthier immune system, and relieves stress (e.g., [10]). In addition to this apparent design element, movement also helps people think better. Movement increases the blood volume in the brain and makes neurons communicate better [14], and this benefit of movement is lifelong; older adults who were engaged in aerobic exercises had enhanced memory abilities over those who were not physically active [2]. Clearly, sitting all day long does not help workers think well. Increasing movement in a workplace benefits productivity. Yet, most leaders give little thought to this physiological truth and necessity and do not promote employee movement throughout the workday, because it looks like employees are not being fully productive. However, the brain simply must have movement to think best. Just standing up and moving for one minute increases blood circulation by $15 \%$ [14].

Given this clear necessity of movement while working, here are a few practical suggestions to foster an active workplace that incorporates movement. First, instead of having printers right next to employees, a leader could place printers a little further away so that employees must stand up and walk a short distance to collect printed material. Second, by allowing walking time throughout a day, a leader encourages employees to move about, ultimately improving their thinking. Third, by promoting group yoga or workout sessions at a workplace, a leader encourages employees to engage in physical activities.

\section{Rest/Break}

Recent brain research found out that even when we are not focusing on a specific work, i.e., "zoning out" or being a "couch potato," our brain is still hard at work [1]. Our brain in these apparent downtimes processes what occurred before and internalizes that experience. This relaxed mind, therefore, is important for organizing thoughts and for creating a later "a-ha!" moment within the employee. Some companies have a "non-working" working day when employees do other things which are not related to work, and it has shown success. For example, Google gives one day a week to allow employees to do something they are interested in, and this practice has resulted in improved overall company productivity [19]. In addition, physical relaxation practice and meditation actually cause enhanced memory [5].

Finally, leaders are encouraged to talk about the importance of sleep and rest. While sleeping, people replay what they did throughout the day. As a result, sleep is critical for assisting in organizing the content of the brain, in unlearning what is unnecessary, and in strengthening memory about what is necessary [16]. Research repeatedly reinforces the idea that those who sleep at least 8 hours experience at least 1.9 as much work productivity as those with less than 5 hours of sleep [6].

\section{Non-monetary Rewards or Incentives}

Most leaders assume that monetary rewards will motivate employees to work harder. Is this commonly held belief really true? Not necessarily. Research study after research study shows that the results are inconclusive in relation to money and job performance. Why is that? Because the brain recognizes money differently from person to person, salary and its motivating influence is very contextual. When money is recognized as an enjoyment, then the reward network in the brain is activated, neurotransmitters such as dopamine are produced, and people feel happy and excited. However, many studies indicate that non-monetary rewards work as well as money - and sometimes even better than money - to motivate many workers. When it comes to motivation, the promise of monetary reward actually reduces intrinsic motivation. Conversely, positive verbal feedback increases intrinsic motivation [4]. Other non-monetary rewards that have a positive impact include higher status, social approval, fairness, and the anticipation of personal growth [19].

One of the main means to trigger the brain's reward network is the establishment and the pursuit of goals. When goals are accomplished, people's reward networks fire up, and this brain response produces feelings of happiness, contentment, and satisfaction. However, a leader needs to be careful about the use of goals, because large and excessively challenging goals can be harmful to employee motivation. The best, brain-based goal strategy is to break goals into small "chunks" and celebrate employee achievement of each goal segment. Spending hours sitting at a desk to complete a specific task continuously simply does not lead to full productivity. Neurotransmitters which are released for a new task are depleted in the brain nerve endings after only about 15 minutes or so [20]. Consequently, a sporadic feeling of accomplishment over completing a small goal might be better and more cost-effective than monetary rewards based on performances.

Finally, giving choices to achieve goals can be a great alternative to monetary incentives. The freedom to choose lets employees feel a sense of ownership and control. Having control is a positive experience for the brain and less of a threat to it. Research shows that providing choices causes the limbic system to react positively. Rather than forcing employees to do a task in a certain fixed way, brain-centered leaders give workers the autonomy to make many decisions themselves. To the brain, this approach is a reward, not a threat. Research indicates that even the perception of having a choice is beneficial for the brain's limbic system, and it reacts positively [13].

\section{Repeat to Remember and Remember to Repeat}

What is the best brain-friendly approach if there is new technology or information that employees must learn? Often, well-meaning leaders schedule an eight-hour training session in a conference room and "require" their employees to 
memorize the new information for later application. Unfortunately, this "sit-and-get" paradigm is not at all supported by current educational research. An improved brain-respecting technique involves repetition, but it is not in a common sense. At its most basic level, repetition has to do with consolidating information. In order to consolidate content in the brain, those facts have to move from shortterm memory into long-term memory. This happens within the hippocampus, the brain's memory center. When a signal enters the brain, it goes first to the cerebral cortex and then to the hippocampus (See Figure 1). However, under normal life conditions, this stored information could take years to become consolidated as a retrievable memory for easy access. Connections among different bits of information associated to a single event or memory has to be strengthened or "solidified" within the brain for those related memories to be usable in any practical, real world way. This memory transition is only accomplished through repetition, and repeated research has shown that only a specific type of repetition accomplishes this necessary transition. As most would agree, cramming for an exam or an extended training in a small meeting room just results in a loss of information within a matter of days.

Researchers have found, however, one method of repetition and review that is consistently superior for memory transfer; that technique is known as spaced repetition. Leaders who want real performance change within their organization should re-expose employees to new information periodically within a certain period of time [8]. While how long the lapse in between sessions has not been fully established by research yet, many research studies seem to indicate that a gap between re-exposures of information of greater than one day is usually preferable (e.g., [3]).

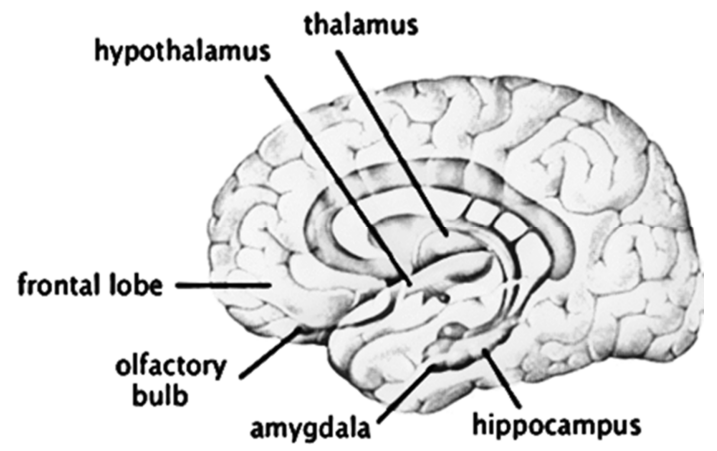

Figure 1. Limbic system. Retrieved from https://courses.lumenlearning.com/boundless-psychology/chapter/structureand-function-of-the-brain/

Additionally, because people have a small capacity of working memory, it is hard to process large quantity of data in a short period of time. Change in behavior is difficult enough for everyone, but learning and synthesizing new information is even more challenging for adults. However, the human brain is malleable, even long after puberty. Given this truth, the leader should make intentional decisions about how new content is introduced in an environment. The primary advice for a leader, then, is an emphasis on "chunking" and repeating over a period of time. Limiting the number of expected tasks is never natural or easy for a leader, but introducing a few manageable tasks over a matter of days or weeks is better for the brain or workers in the long run. Giving focused fifteen-minute attention on one new piece of information is preferable to giving shallow one hour presentations on several new pieces of information. Focus is the key, and with all other information that employees interact with these days (e.g., emails, texts, etc.), a small chunk of information is all that can be reasonably handled, and then repeating this focused content over time enhances memory and improves real learning [20].

\section{Emotional Shortcuts, "Gut Feelings"}

Modern brain research teaches that unconscious thinking is more important than previously known. Most tend to think that logical and conscious decisions are superior to unconscious and illogical "emotions;" however, prehistoric ancestors of modern man relied heavily on their emotions and primitive thinking (instinctual behavior) when they were threatened. As a result, when a life-or-death situation arises, the human body reacts and triggers appropriate physiological responses (e.g., sweating, increased blood pressure, shallow breathing, etc.). Because of this evolutionary training, the brain recognizes the threat before it consciously registers, and commonly, people think of this immediate, unconscious response as "having a hunch" or a "gut feeling." Researchers speculate that modern man uses less than $10 \%$ of conscious thinking; the remaining time is involved with unconscious thought. The brain processes information all the time, verifying incoming input with previous experience, and it makes predictions about what is next. In order to give more time to process more challenging information, the brain uses a shortcut to address "simple" events [15]. The decisionmaking process within the brain to determine whether to devote more time and concentration to a topic or whether to leave that information to instinct happens so quickly within the brain that the individual is not usually aware of it, and this action feels to the person like a "gut feeling." As expected, the more a person experiences a certain subject or event, the more accurate his or her apparent "hunches" become. This is because $\mathrm{s} /$ he has more information to match with from the past. Gut feelings are doubted and rejected easily in logic-prevalent, data-driven workplaces, but more and more neurological research findings suggest that the hunches should not be ignored; they should be discussed and evaluated and may lead to making better decisions [19].

\section{Conclusion}

The six principles presented above - positive environment, movement, rest, non-monetary reward, repetition, and using gut feelings - are not at all difficult to achieve in the workplace. Leaders who have not yet done so are encouraged to take small steps toward fostering a brain friendly workplace. Merely keeping these principles in mind 
throughout the workday planning process will help employees achieve better results. In addition, leaders are encouraged to explain to employees how their brains work, giving them an edge on improving their own work. Brain research like that presented above is yet another modern tool to assist leaders in accomplishing the most they possibly can for their organizations.

\section{Statement}

The attached manuscript has never been published and is not currently under review elsewhere.

\section{References}

[1] Baird, B., Smallwood, J., Mrazek, M., Kam, J., Franklin, M., \& Schooler, J. (2012). Inspired by Distraction: Mind Wandering Facilitates Creative Incubation. Psychological Science, 23 (10), 1117-1122.

[2] Brinke, L., Bolandzadeh, N., Nagamatsu, L., Hsu, C., Davis, J., Miran-Khan, K., \& Liu-Ambrose, T. (2014). Aerobic exercise increases hippocampal volume in older women with probable mild cognitive impairment: a 6-month randomized controlled trial. British Journal of Sports Medicine, 49, 248254 .

[3] Cepeda, N., Coburn, N., Rohrer, D., Wixted, J., Mozer, M., \& Pashler, H. (2009). Optimizing distributed practice: theoretical analysis and practical implications. Experimental Psychology, $56,236-246$.

[4] Deci, E. (1971). Effects of externally mediated rewards on intrinsic motivation. Journal of Personality and Social Psychology, 18 (1), 105-115.

[5] Flor, K., Monir. C., Bita, A., \& Shahnaz. N. (2013). Effect of relaxation training on working memory capacity and academic achievement in adolescents. Procedia-Social and Behavioral Sciences, 82, 608-613.

[6] Gingerich, S., Seaverson, E., \& Anderson, D. (2018). Association between sleep and productivity loss among 598, 676 employees from multiple industries. American Journal of Health Promotion, 32 (4), 1091-1094.

[7] Gordon, E., \& Williams, L. (2008). An integrative neuroscience platform: Applications to profiles of negativity and positivity bias. Journal of Integrative Neuroscience, 7 (3), 345-366.
[8] Karpicke, J., \& Roediger, H., III (2007). Expanding retrieval practice promotes short-term retention, but equally spaced retrieval enhances long-term retention. Journal of Experimental Psychology: Learning, Memory, and Cognition, 33, 704-719.

[9] Keysers, C., \& Gazzola, V. (2006). Towards a unifying neural theory of social cognition. Progress in Brain Research, 156, 379-401.

[10] Kohll, A. (2019, January 09). Why we pay our employees to exercise at work. Forbes. Retrieved from https://www.forbes.com/sites/alankohl1/2019/01/09/why-wepay-our-employees-to-exercise-at-work/

[11] LeDoux, J. (1996). The Emotional Brain. New York: Houghton Mifflin.

[12] Medina, J. (2010). Brain Rules: 12 Principles for Surviving and Thriving at Work, Home, and School. Seattle, WA: Pear Press.

[13] Rock, D. (2009). Your brain at work: Strategies for Overcoming Distraction, Regaining Focus, and Working Smarter All Day Long. New York, NY: Harpers Collins Publishers.

[14] Sausa, D. (2011). How The Brain Learns. 4th ed. Thousand Oaks, CA: Corwin Press.

[15] Soon, C., Brass, M., Heinze, H., \& Haynes, J. (2008). Unconscious determinants of free decisions in the human brain. Nature Neuroscience, 11, 543-545.

[16] Stickergold, R. \& Walker, M. (2007). Sleep-dependent memory consolidation and reconsolidation. Sleep Medicine, 8 , 331-343.

[17] Tabibnia, G., \& Liberman, M. (2007). Fairness and cooperation are rewarding: Evidence from social cognitive neuroscience. Annals of New York Academy of Science, 1118, 90-101.

[18] Turturici, D. (2019, October 18). Neuroscience Sheds New Light on Change Management Strategies. Retrieved from https://www.bpminstitute.org/resources/articles/neurosciencesheds-new-light-on-change-management-strategies

[19] Waytz, A., \& Mason, M. (2013). Your brain at work. What a new approach to neuroscience can teach us about management. Harvard Business Review, 91 (7-8), 102-111.

[20] Willis, J. (2010). Current Impact of Neuroscience in Teaching and Learning. In D. Sousa (Ed.), Mind, Brain, and Education: Neuroscience implication for the classroom (pp. 45-66). Bloomington, IN: Solution Tree Press. 